\title{
Altered functions of alveolar macrophages and NK cells involved in asbestos-related diseases
}

\author{
Yasumitsu Nishimura $\cdot$ Megumi Maeda $\cdot$ Naoko Kumagai-Takei $\cdot$ \\ Suni Lee $\cdot$ Hidenori Matsuzaki $\cdot$ Yasuhiko Wada Tamako Nishiike-Wada • \\ Hiroshi Iguchi $\cdot$ Takemi Otsuki
}

Received: 15 December 2012/ Accepted: 12 February 2013/Published online: 6 March 2013

(C) The Japanese Society for Hygiene 2013

\begin{abstract}
Asbestos exposure causes asbestosis and malignant mesothelioma, disorders which remain difficult to cure. We focused on alveolar macrophages (AM) and natural killer (NK) cells in asbestosis and mesothelioma, respectively, and examined their functions upon exposure to asbestos or in patients with mesothelioma. Exposure to asbestos caused rat $\mathrm{AM}$ to exhibit high production of transforming growth factor-beta (TGF- $\beta$ ) with prolonged survival in the absence of other cells, not simultaneously with the apoptosis caused by asbestos. The NK cell line showed impaired cytotoxicity with altered expression of activating receptors upon exposure to asbestos, and primary NK cells in culture with asbestos and peripheral blood NK cells in mesothelioma shared a decrease in expression of NKp46, a representative activating receptor. The AM finding indicates that AM contribute to asbestosis
\end{abstract}

Y. Nishimura $(\varangle) \cdot$ N. Kumagai-Takei · S. Lee ·

H. Matsuzaki · T. Otsuki

Department of Hygiene, Kawasaki Medical School,

577 Matsushima, Kurashiki 701-0192, Japan

e-mail: yas@med.kawasaki-m.ac.jp

\section{Maeda}

Department of Biofunctional Chemistry, Division

of Agricultural and Life Science, Graduate School

of Natural Science and Technology, Okayama University,

Okayama, Japan

Y. Wada

Faculty of Nutrition, University of Kochi, Kochi, Japan

T. Nishiike-Wada

Department of Life Design, Otemae College, Itami, Japan

H. Iguchi

Department of Health and Nutrition, Faculty of Human Life,

Jin-ai University, Echizen, Japan by playing a direct role in the fibrogenic response, as well as the inflammatory response. The response of NK cells indicates that exposure to asbestos has an immune-suppressive effect, as well as a tumorigenic effect. Our studies therefore reveal novel effects of asbestos exposure on AM and tumor immunity, which may represent valuable information for construction of a strategy for prevention and cure of asbestosis and malignant mesothelioma.

Keywords Asbestos - Alveolar macrophage .

Natural killer cell · Asbestosis · Malignant mesothelioma

\section{Introduction}

Asbestos exists in the form of naturally occurring fibrous minerals such as chrysotile, crocidolite, and amosite, all of which consist of silicon, oxygen, hydrogen, and various metal cations. Asbestos has been utilized globally to make various products such as heat-resistant clothes, tiles, and walls, because asbestos consists of thin, long fibers with high flexibility and strong resistance to fire and heat. However, it is now known that exposure to asbestos causes two serious health disorders that cannot be cured at present. One of these disorders is asbestosis, a form of lung fibrosis following chronic inflammation caused by inhalation of asbestos. The other disorder is malignant mesothelioma, comprising a tumor of mesothelial cells, mainly in the pleural or occasionally the peritoneal cavity, caused by inhalation of asbestos. Therefore, we focused on examination of immune-competent cells that may contribute to development of asbestosis and mesothelioma if their functions are altered upon exposure to asbestos. The targets for asbestosis and mesothelioma were alveolar macrophages (AM) and natural killer (NK) cells, respectively. 
Here, we review our studies regarding asbestos-caused functional alteration of AM and NK cells. For the latter, we show the functional similarity between NK cells in culture upon exposure to asbestos and those in mesothelioma patients. These studies have resulted in new insights concerning the development of asbestosis and malignant mesothelioma.

\section{Role of alveolar macrophages in lung inflammation caused by asbestos exposure}

Asbestosis is a form of lung fibrosis following inflammation caused by inhalation of asbestos. It is well known that AM play a crucial role in inflammation caused by exposure to asbestos. AM produce reactive oxygen species (ROS) [1], reactive nitrogen species (RNS) [2, 3], as well as chemoattractant and inflammatory cytokines including tumor necrosis factor-alpha (TNF- $\alpha$ ), interleukin (IL)-1 $\beta$, and IL-6 [4, 5]. Since inhaled asbestos accumulates in the lungs, inflammation caused by asbestos continues chronically. Therefore, these ROS/RNS-dependent inflammatory responses lead to repeated injury of lung tissue followed by tissue repair. As a consequence, a large amount of extracellular matrix (ECM) is produced, and the lungs reach a state of fibrosis and asbestosis [6, 7]. In particular, TNF- $\alpha$ plays a role in production of transforming growth factor-beta
(TGF)- $\beta$, which induces production of ECM, and previous studies have demonstrated that $\mathrm{TNF}-\alpha$ receptor knockout mice showed decreases in production of TGF- $\beta$ and accumulation of ECM after exposure to asbestos [6, 8]. Moreover, asbestos exposure causes apoptosis of lung epithelial cells, mesothelial cells, and AM [9], and it has been reported that intratracheal instillation with apoptotic cells of macrophages induces increased production of TGF- $\beta$ and fibrosis of the lungs [10]. Thus, it has been shown that asbestos-caused lung fibrosis is dependent on the overproduction of ECM and that AM indirectly contribute to the development of lung fibrosis, in which AM play a role in the inflammatory response prior to production of TGF- $\beta$, leading to a fibrogenic response (Fig. 1, left).

\section{Long-surviving alveolar macrophages with high production of TGF- $\beta$ caused by exposure to asbestos}

As mentioned above, AM are thought to contribute to inflammation and the generation of apoptotic cells after exposure to asbestos. However, AM are also able to produce TGF- $\beta$, which raises the possibility that AM might contribute more directly to the development of lung fibrosis through TGF- $\beta$ production. Therefore, we examined alteration in functions of AM caused by exposure to asbestos using in vivo and in vitro experiments. We first

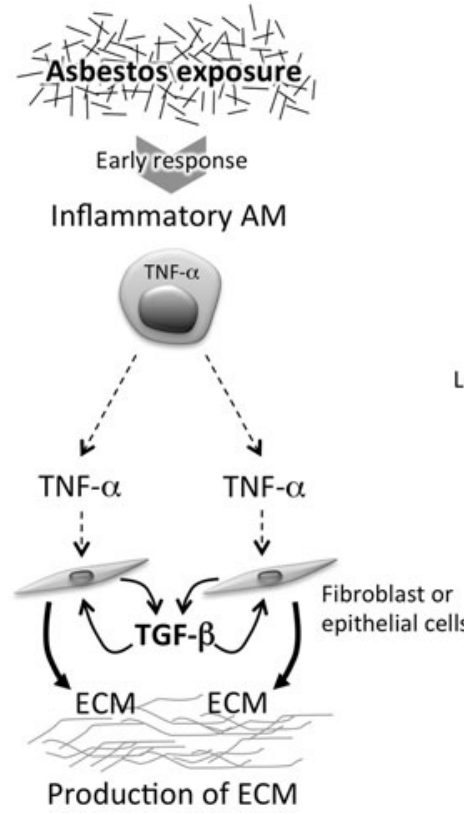

Fig. 1 Asbestos exposure-induced generation of fibrogenic alveolar macrophages with high production of TGF- $\beta$. The illustration shows the more significant contribution of alveolar macrophages (AM) to asbestos-caused lung fibrosis by production of TGF- $\beta$, in addition to production of inflammatory cytokines. TNF- $\alpha$ produced by AM early after exposure to asbestos stimulates fibroblasts or lung epithelial
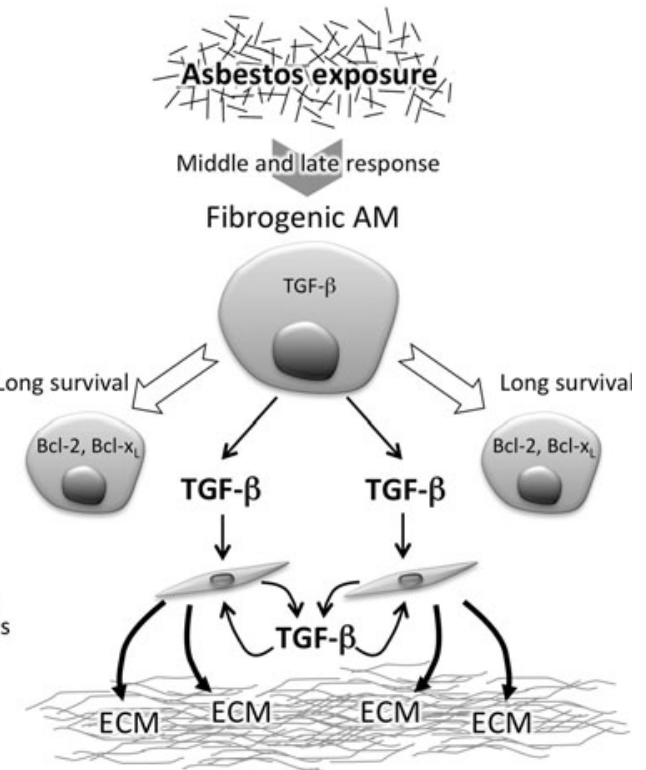

Accumulated production of ECM

cells to produce TGF- $\beta$, leading to production of extracellular matrix (ECM) (left). However, as demonstrated by our studies, asbestos exposure induces generation of fibrogenic AM with high production of TGF- $\beta$ and enhanced survival. It is probable that these AM contribute to accumulated production of ECM by their long-lasting production of TGF- $\beta$ in addition to an inflammatory response (right) 
tried to confirm the inflammatory response and subsequent production of TGF- $\beta$ in the lungs following exposure to asbestos. Wistar rats were instilled with $4 \mathrm{mg}$ of chrysotile $\mathrm{B}(\mathrm{CB})$ via the trachea, and bronchoalveolar lavage fluid (BALF) was then obtained from these rats. BALF obtained 1 day after instillation with $\mathrm{CB}$ showed increases in granulocytes and inflammatory cytokines, TNF- $\alpha$, IL-1 $\beta$, and IL-6, compared with the BALF of control rats. Five days after instillation with $\mathrm{CB}, \mathrm{TGF}-\beta$ increased in the BALF, in which many apoptotic cells were found. We then examined the production of TGF- $\beta$ by AM prepared from the BALF of CB-instilled rats. These results demonstrate that we constructed an animal model for the inflammatory response and subsequent production of TGF- $\beta$ following exposure to asbestos. Therefore, AM prepared from the BALF of CB-instilled or control rats were cultured with or without $\mathrm{CB}$ for 5 days, and examined for the production of TGF- $\beta$. AM from CB-instilled rats showed high production of TGF- $\beta$, which was not augmented by the in vitro exposure to $\mathrm{CB}$. In contrast, $\mathrm{AM}$ from control rats showed low production of TGF- $\beta$. However, in vitro exposure to CB caused an increase in TGF- $\beta$ production by AM to the same level observed for AM from $\mathrm{CB}$-instilled rats, following high production of TNF- $\alpha$ at 1 day after culture. We then examined apoptosis of AM in the culture. AM actually showed apoptosis upon exposure to a high dose of $\mathrm{CB}$ at 12.5 or $25 \mu \mathrm{g} / \mathrm{cm}^{2}$, but showed no such response at 1.25 or $2.5 \mu \mathrm{g} / \mathrm{cm}^{2}$. In contrast, the production of TGF- $\beta$ by $\mathrm{AM}$ peaked at $2.5 \mu \mathrm{g} / \mathrm{cm}^{2}$ of $\mathrm{CB}$, but did not increase following exposure to over $5.0 \mu \mathrm{g} / \mathrm{cm}^{2}$. Moreover, AM showed further increased production of TGF- $\beta$ in the subsequent culture for an additional 3 days, and also showed increased expression of $B c l-2$ and $B c l-x_{L}$, genes related to cell survival [11]. These results indicate that AM acquire a high level of TGF- $\beta$ production with enhanced survival following the inflammatory response upon exposure to asbestos, in which cells other than AM were not necessary (Fig. 1, right). In addition, it was found that high production of TGF- $\beta$ by AM and their apoptosis are induced upon exposure to different doses of CB. These new findings allow us to consider the possibility that such "fibrogenic" AM with high production of TGF- $\beta$ and enhanced survival may contribute to an early stage of asbestos-caused lung fibrosis by moving around, producing TGF- $\beta$, and living for a long period.

\section{Malignant mesothelioma and antitumor immunity}

It is well known that malignant mesothelioma is caused by inhalation of asbestos [12, 13]. In previous reports, it was shown that levels of oxidized pyrimidines and alkylated bases are strongly correlated with the length of occupational exposure to asbestos, and that an increase in the mutation frequency of lung DNA was induced by instillation with asbestos via the trachea $[14,15]$. Based on these findings, most researchers think that malignant mesothelioma results exclusively from the tumorigenic effect of exposure to asbestos. However, there are some interesting issues concerning the relation between mesothelioma and asbestos exposure. People who have previously inhaled asbestos take about 40 years to develop malignant mesothelioma. In addition, it is known that malignant mesothelioma more often develops in people exposed to a low or moderate dose of asbestos rather than a high dose, which is inconsistent with the general rule concerning the effect of toxic materials. These findings support the possibility that the development of malignant mesothelioma may be related to alternative effects of asbestos exposure other than the tumorigenic effect, one of which is antitumor immunity. In the body, antitumor immunity plays a role in surveillance and deletion of transformed cells or early tumor cells, in which NK cells act at the front line of tumor immunity as first effectors to combat cytotoxicity induced by the abnormal cells. Therefore, we examined the effects of asbestos exposure on cytotoxicity of NK cells, and also examined the character of NK cells in patients with malignant mesothelioma.

\section{NK cell receptors and cytotoxicity of NK cells}

Unlike T cells, NK cells utilize various kinds of receptors to recognize target cells. These receptors are divided into activating and inhibitory receptors, which respectively transduce and inhibit signals, connected to exocytosis of cytotoxic granules for target cells [16]. The granules include perforin and granzymes, which make targets undergo apoptosis [17]. Thus, the cytotoxicity of NK cells is controlled by a balance between signals derived from activating and inhibitory receptors. NKG2D is the most well-known NK cell-activating receptor, and a member of the NKG2 family, which is characterized by a lectin-like domain [18]. The NKG2 family also includes members with an inhibitory function such as NKG2A, which makes heterodimers with CD94 that act as inhibitory receptors. Natural cytotoxicity receptors (NCRs) and the signaling lymphocytic activation molecule (SLAM) family of receptors are also other groups of NK cell-activating receptors. NKp46 is a representative member of NCRs expressed on NK cells constitutively [16, 19]. NCRs, including others such as NKp44 and NKp30, also play a major role in the NK-mediated killing of target cells. 2B4 is also expressed on NK cells, and can induce cytotoxicity of NK cells by engagement with CD48, the ligand for 2B4, or the anti-2B4 antibody [20-22]. The signals from those 
NK cell-activating receptors are mediated by common molecules, one of which is extracellular signal-regulated kinase (ERK) [23]. It has been shown that ERK is essential for the polarization of cytotoxic granules to the immunological synapse, the area in contact with target cells [24]. Therefore, we focused on NK cell receptors and examined the effect of exposure to asbestos on cytotoxicity and receptor expression of NK cells, followed by analysis of the phosphorylation of ERK. In addition, peripheral blood NK cells in patients with malignant mesothelioma were examined and compared with those of healthy volunteers. Finally, we clarified that NK cells in mesothelioma patients showed a similar character of impaired cytotoxicity with altered expression of NK cell-activating receptors.

\section{Impaired cytotoxicity with altered expression of NK cell-activating receptors related to malignant mesothelioma as well as asbestos exposure}

We initially started the long-term culture of a human NK cell line, YT-A1, upon exposure to CB asbestos at $5 \mu \mathrm{g} / \mathrm{ml}$, which did not show any influence on cell growth or apoptosis of YT-A1 cells. The CB-exposed subline, YT-CB5, was periodically examined for cytotoxicity against K562 cells and expression of NK cell receptors and compared with the control line, YT-Org. YT-CB5 did not differ in cytotoxicity from YT-Org 1 month after culture with CB asbestos. However, it showed a decrease in cytotoxicity after around 5 months of culture, accompanied with decreased expression of NKG2D and 2B4, but not NKp46 [25]. In contrast, YT-CB5 showed only a slight change in expression levels of NKG2A and CD94. In agreement with these results, YT-CB5 showed a decrease in degranulation induced by stimulation with antibodies to NKG2D or $2 \mathrm{~B} 4$. In addition, phosphorylation of ERK induced by stimulation with NKG2D was lower in YT-CB5 than in YT-Org [26]. To confirm the relationship among cytotoxicity, expression of NK cell-activating receptors, and phosphorylation of ERK in primary cells, peripheral blood NK cells from healthy volunteers were examined and compared. NK cells with high cytotoxicity showed high expression of NKG2D or NKp46, but not 2B4, and high phosphorylation of ERK, whereas the opposite was shown by NK cells with low cytotoxicity. On the basis of these results, we examined peripheral blood NK cells in patients with malignant mesothelioma. The $\mathrm{CD}^{-}{ }^{-} \mathrm{CD} 56^{+} \mathrm{NK}$ cells of these patients showed impaired cytotoxicity with altered expression of NK cell-activating receptors, in which a decrease was observed for NKp46 but not for NKG2D or 2B4 [25]. Since the decrease in expression of NKp46 was not observed in the experiment using the cell line, we tried to culture primary cells upon exposure to asbestos. Peripheral blood mononuclear cells (PBMCs) were cultured with $\mathrm{CB}$ asbestos at $5 \mu \mathrm{g} / \mathrm{ml}$ for 7 days, and $\mathrm{CD}^{-}{ }^{-} \mathrm{CD} 56^{+} \mathrm{NK}$ cells in harvested PBMCs were examined for expression of activating receptors. As found for NK cells in mesothelioma patients, NK cells in the culture with $\mathrm{CB}$ showed decreased expression of NKp46, but not NKG2D or $2 \mathrm{~B} 4$. In contrast, exposure to glass wool, a representative asbestos substitute of manmade mineral fibers, did not cause a decrease in NKp46. These results indicate that exposure to asbestos causes impairment in cytotoxicity of NK cells with altered expression of activating receptors, and that the decrease in expression of NKp46 in NK cells is related to asbestos exposure and malignant mesothelioma. These findings suggest that such immunological effects resulting from exposure to asbestos might contribute to the development of mesothelioma and its tumorigenic effect.

\section{Conclusions}

We investigated functional alterations resulting from exposure to asbestos in two kinds of immune cells related to asbestosis and malignant mesothelioma. In the former investigation, our study demonstrated that $\mathrm{AM}$ have the potential to augment their own production of TGF- $\beta$ upon exposure to asbestos in an independent manner, not simultaneously with the apoptosis of AM caused by asbestos exposure. The contribution of AM to asbestosis is usually explained as an inflammatory response, in which AM produce inflammatory cytokines and ROS/RNS upon exposure to asbestos, which subsequently leads to the production of TGF- $\beta$ followed by accumulation of ECM in the lungs. However, as shown in our study, asbestos exposure causes AM to produce a high amount of TGF- $\beta$ with prolonged survival, which is not related to any other types of cells such as lung epithelial cells or fibroblasts. Although the amount of TGF- $\beta$ produced by AM may not be large, the finding concerning autologous acquisition of TGF- $\beta$ production by AM cannot be ignored because TGF- $\beta$ induces more TGF- $\beta$ production in a positive feedback regulation, which amplifies signals to a level sufficient for biological activity [27-30]. It is possible that such a fibrogenic type of AM might move around and produce TGF- $\beta$ over the lungs upon exposure to asbestos, in which accelerated production of TGF- $\beta$ might be triggered. As illustrated in Fig. 1, the increased signals of TGF- $\beta$ provided by AM may lead to a more severe situation of ECM accumulation, compared with the situation in which AM just produce inflammatory cytokines. Furthermore, it is interesting that our results showed that the generation of fibrogenic AM was separated from the asbestos-caused apoptosis of cells, implying the silent generation of fibrogenic AM without being accompanied by an obvious 
inflammatory response upon exposure to asbestos. Thus, our findings regarding AM emphasize their role in the fibrogenic response caused by exposure to asbestos, and provide additional information concerning the mechanism of asbestosis that may be useful for the development of an effective clinical strategy against this disorder.

In the latter study, we demonstrated that asbestos exposure causes impaired cytotoxicity of NK cells with altered expression of activating receptors. We also showed that NK cells in culture upon exposure to asbestos and NK cells in patients with malignant mesothelioma show the same decreased expression of NKp46. Although the results obtained from experiments with the NK cell line were not consistent with those from the primary NK cell culture or from mesothelioma patients, they are significant because they revealed the asbestos exposure-caused alteration in expression of NK cell-activating receptors and the relationship between decreased expression of receptor and subsequent signal transduction. It is known that NK cells are rich in nonlymphoid organs, including the lung and liver, as well as lymphoid organs, which may suggest that NK cells are mainly exposed to asbestos in the lungs. However, many studies have shown the existence of asbestos bodies, fibers of asbestos coated with an iron-rich material, in regional lymph nodes [31, 32]. It has also been reported that nonoccupationally and occupationally asbestos-exposed individuals have asbestos in their lymph nodes
[33-35]. Therefore, it is probable that NK cells suffer the harmful effects of asbestos in lymph nodes as well as in the lungs. In particular, the most noteworthy finding is that the pattern of alteration in expression of activating receptors was very similar between NK cells in culture exposed to asbestos and NK cells in patients with malignant mesothelioma, in which a decrease was observed for NKp46 but not for NKG2D or 2B4. Consideration of these findings together with the observation of no decrease in NKp46 upon exposure to glass wool suggests that impaired cytotoxicity of NK cells with decreased NKp46 in mesothelioma might be related to the effect of asbestos inhaled and accumulated in the body. Such an immune-suppressive effect of asbestos exposure might contribute to the development of malignant mesothelioma with its tumorigenic effect.

Thus, we present new findings concerning asbestoscaused functional alteration in AM and NK cells in relation to asbestosis and malignant mesothelioma, respectively. The findings concerning AM emphasize the fact that AM are the primary cells that phagocytose inhaled asbestos in the lungs. The alteration in function of AM upstream of the asbestos-caused response may be an event that seeds lung fibrosis. Results regarding the functions of NK cells demonstrate the presence of the suppressive effect of asbestos exposure on antitumor immunity. In fact, we also showed that $\mathrm{T}$ helper cells in culture with asbestos and in patients
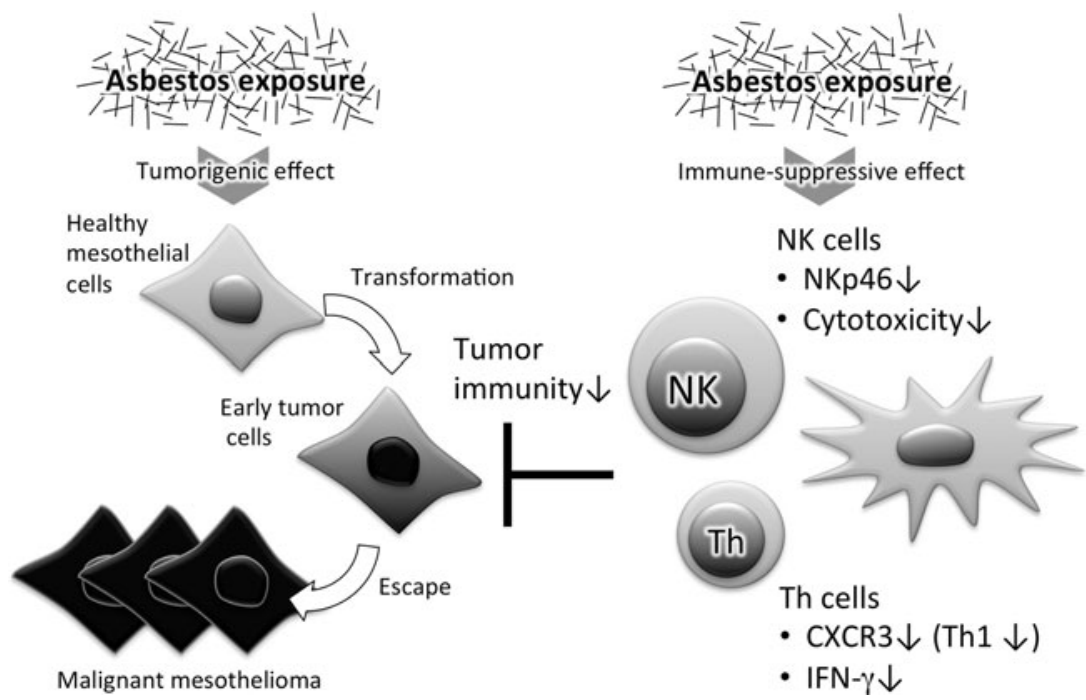

Fig. 2 Immune-suppressive effect of asbestos exposure. The illustration shows that asbestos exposure has the potential to impair the functions of $\mathrm{NK}$ and $\mathrm{Th}$ cells, in addition to the previous findings concerning the tumorigenic effect of asbestos. As demonstrated by our studies, asbestos exposure causes decreased cytotoxicity of NK cells with alteration in expression of activating receptors. In particular, the decrease in expression of NKp46 is shared among primary NK cells in peripheral blood mononuclear cell (PBMC) culture upon exposure to asbestos and NK cells in the peripheral

blood of patients with malignant mesothelioma. Furthermore, our studies demonstrated decreases in CXCR3 expression and interferongamma (IFN- $\gamma$ ) production by Th cells upon exposure to asbestos, which was also observed in mesothelioma patients. Our findings suggest that these immune-suppressive effects of asbestos exposure might allow early tumor cells to escape from tumor immunity, leading to development of malignant mesothelioma in people exposed to asbestos 
with mesothelioma showed decreased Th1 cell activity (Fig. 2) [36, 37]. The immune system originally plays a role in deletion of exogenous or endogenous nonself substances to maintain health. As shown in our studies, exposure to asbestos may interfere with these immune responses and thereby lead to health disorders. Future study of the immunological effects of asbestos exposure may provide valuable information to solve the health problems caused by asbestos.

Acknowledgments We thank Ms. Tamayo Hatayama, Shoko Yamamoto, Minako Kato, and Naomi Miyahara for their technical help.

Conflict of interest None of the authors has a financial relationship with a commercial entity that has an interest in the subject of this manuscript.

\section{References}

1. Kamp DW, Graceffa P, Pryor WA, Weitzman SA. The role of free radicals in asbestos-induced diseases. Free Radic Biol Med. 1992;12:293-315.

2. Iguchi H, Kojo S, Ikeda M. Nitric oxide (NO) synthase activity in the lung and NO synthesis in alveolar macrophages of rats increased on exposure to asbestos. J Appl Toxicol JAT. 1996;16: 309-15.

3. Nishiike T, Nishimura Y, Wada Y, Iguchi H. Production of nitric oxide elevates nitrosothiol formation resulting in decreased glutathione in macrophages exposed to asbestos or asbestos substitutes. Arch Toxicol. 2005;79:83-9.

4. Dubois CM, Bissonnette E, Rola-Pleszczynski M. Asbestos fibers and silica particles stimulate rat alveolar macrophages to release tumor necrosis factor. Autoregulatory role of leukotriene B4. Am Rev Respir Dis. 1989;139:1257-64.

5. Li XY, Lamb D, Donaldson K. The production of TNF-alpha and IL-1-like activity by bronchoalveolar leucocytes after intratracheal instillation of crocidolite asbestos. Int $\mathrm{J}$ Exp Pathol. 1993;74:403-10.

6. Liu JY, Brody AR. Increased TGF-beta1 in the lungs of asbestosexposed rats and mice: reduced expression in TNF-alpha receptor knockout mice. J Environ Pathol Toxicol Oncol Off Organ Int Soc Environ Toxicol Cancer. 2001;20:97-108.

7. Mossman BT, Churg A. Mechanisms in the pathogenesis of asbestosis and silicosis. Am J Respir Crit Care Med. 1998;157: 1666-80.

8. Liu JY, Brass DM, Hoyle GW, Brody AR. TNF-alpha receptor knockout mice are protected from the fibroproliferative effects of inhaled asbestos fibers. Am J Pathol. 1998;153:1839-47.

9. Hamilton RF, Iyer LL, Holian A. Asbestos induces apoptosis in human alveolar macrophages. Am J Physiol. 1996;271:L813-9.

10. Wang L, Antonini JM, Rojanasakul Y, Castranova V, Scabilloni JF, Mercer RR. Potential role of apoptotic macrophages in pulmonary inflammation and fibrosis. J Cell Physiol. 2003;194:215-24.

11. Nishimura Y, Nishiike-Wada T, Wada Y, Miura Y, Otsuki T, Iguchi $\mathrm{H}$. Long-lasting production of TGF-beta1 by alveolar macrophages exposed to low doses of asbestos without apoptosis. Int J Immunopathol Pharmacol. 2007;20:661-71.

12. Mossman BT, Kamp DW, Weitzman SA. Mechanisms of carcinogenesis and clinical features of asbestos-associated cancers. Cancer Invest. 1996;14:466-80.
13. Sporn TA, Roggli VL. Mesothelioma. In: Roggli VL, Oury TD, Sporn TA, editors. Pathology of asbestos-associated diseases. New York: Springer; 2004. p. 104-68.

14. Dusinska M, Collins A, Kazimirova A, Barancokova M, Harrington V, Volkovova $\mathrm{K}$, et al. Genotoxic effects of asbestos in humans. Mutat Res. 2004;553:91-102.

15. Topinka J, Loli P, Georgiadis P, Dusinska M, Hurbankova M, Kovacikova Z, et al. Mutagenesis by asbestos in the lung of lambda-lacI transgenic rats. Mutat Res. 2004;553:67-78.

16. Moretta A, Bottino C, Vitale M, Pende D, Cantoni C, Mingari $\mathrm{MC}$, et al. Activating receptors and coreceptors involved in human natural killer cell-mediated cytolysis. Annu Rev Immunol. 2001;19:197-223.

17. Trapani JA, Smyth MJ. Functional significance of the perforin/ granzyme cell death pathway. Nat Rev Immunol. 2002;2:735-47.

18. Yokoyama WM, Plougastel BF. Immune functions encoded by the natural killer gene complex. Nat Rev Immunol. 2003;3: 304-16.

19. Sivori S, Pende D, Bottino C, Marcenaro E, Pessino A, Biassoni $\mathrm{R}$, et al. NKp46 is the major triggering receptor involved in the natural cytotoxicity of fresh or cultured human NK cells. Correlation between surface density of NKp46 and natural cytotoxicity against autologous, allogeneic or xenogeneic target cells. Eur J Immunol. 1999;29:1656-66.

20. Endt J, Eissmann P, Hoffmann SC, Meinke S, Giese T, Watzl C. Modulation of 2B4 (CD244) activity and regulated SAP expression in human NK cells. Eur J Immunol. 2007;37:193-8.

21. Garni-Wagner BA, Purohit A, Mathew PA, Bennett M, Kumar V. A novel function-associated molecule related to non-MHCrestricted cytotoxicity mediated by activated natural killer cells and T cells. J Immunol. 1993;151:60-70.

22. Valiante NM, Trinchieri G. Identification of a novel signal transduction surface molecule on human cytotoxic lymphocytes. J Exp Med. 1993;178:1397-406.

23. Chen X, Trivedi PP, Ge B, Krzewski K, Strominger JL. Many NK cell receptors activate ERK2 and JNK1 to trigger microtubule organizing center and granule polarization and cytotoxicity. Proc Natl Acad Sci USA. 2007;104:6329-34.

24. Chen X, Allan DS, Krzewski K, Ge B, Kopcow H, Strominger JL. CD28-stimulated ERK2 phosphorylation is required for polarization of the microtubule organizing center and granules in YTS NK cells. Proc Natl Acad Sci USA. 2006;103:10346-51.

25. Nishimura Y, Miura Y, Maeda M, Kumagai N, Murakami S, Hayashi $\mathrm{H}$, et al. Impairment in cytotoxicity and expression of NK cell- activating receptors on human NK cells following exposure to asbestos fibers. Int J Immunopathol Pharmacol. 2009;22:579-90.

26. Nishimura Y, Maeda M, Kumagai N, Hayashi H, Miura Y, Otsuki T. Decrease in phosphorylation of ERK following decreased expression of NK cell-activating receptors in human NK cell line exposed to asbestos. Int J Immunopathol Pharmacol. 2009;22: 879-88.

27. Bascom CC, Wolfshohl JR, Coffey RJ Jr, Madisen L, Webb NR, Purchio AR, et al. Complex regulation of transforming growth factor beta 1 , beta 2 , and beta 3 mRNA expression in mouse fibroblasts and keratinocytes by transforming growth factors beta 1 and beta 2. Mol Cell Biol. 1989;9:5508-15.

28. Kim SJ, Angel P, Lafyatis R, Hattori K, Kim KY, Sporn MB, et al. Autoinduction of transforming growth factor beta 1 is mediated by the AP-1 complex. Mol Cell Biol. 1990;10:1492-7.

29. Miyazono K. Positive and negative regulation of TGF-beta signaling. J Cell Sci. 2000;113(Pt 7):1101-9.

30. O'Reilly MA, Danielpour D, Roberts AB, Sporn MB. Regulation of expression of transforming growth factor-beta 2 by transforming growth factor-beta isoforms is dependent upon cell type. Growth Factors. 1992;6:193-201. 
31. Godwin MC, Jagatic J. Asbestos and mesotheliomas. Environ Res. 1970;3:391-416.

32. Roggli VL, Benning TL. Asbestos bodies in pulmonary hilar lymph nodes. Modern Pathol Off J US Can Acad Pathol Inc. 1990;3:513-7.

33. Dodson RF, Huang J, Bruce JR. Asbestos content in the lymph nodes of nonoccupationally exposed individuals. Am J Ind Med. 2000;37:169-74.

34. Dodson RF, Williams MG Jr, Corn CJ, Brollo A, Bianchi C. Asbestos content of lung tissue, lymph nodes, and pleural plaques from former shipyard workers. Am Rev Respir Dis. 1990;142:843-7.
35. Dodson RF, Williams MG Jr, Corn CJ, Brollo A, Bianchi C. A comparison of asbestos burden in lung parenchyma, lymph nodes, and plaques. Ann N Y Acad Sci. 1991;643:53-60.

36. Maeda M, Nishimura Y, Hayashi H, Kumagai N, Chen Y, Murakami S, et al. Decreased CXCR3 expression in CD4+ T cells exposed to asbestos or derived from asbestos-exposed patients. Am J Respir Cell Mol Biol. 2011;45:795-803.

37. Maeda M, Nishimura Y, Hayashi H, Kumagai N, Chen Y, Murakami S, et al. Reduction of CXC chemokine receptor 3 in an in vitro model of continuous exposure to asbestos in a human T-cell line, MT-2. Am J Respir Cell Mol Biol. 2011;45:470-9. 